Pacific Journal of Mathematics

IRREDUCIBLE CONGRUENCE RELATIONS ON LATTICES 


\section{IRREDUCIBLE CONGRUENCE RELATIONS ON LATTICES}

\section{T. FINKBEINER}

1. Introduction. The structure of the lattice $L$ is known to depend upon properties of the distributive lattice $\vartheta(L)$ of all congruence relations on $L$; for example,

(1.1) (Birkhoff [1]) $L$ is a subdirect union of a finite number of simple lattices if and only if $\vartheta(L)$ is a finite Boolean algebra,

(1.2) (Dilworth [2]) $L$ is a direct union of a finite number of simple lattices if and only if $\vartheta(L)$ is a finite Boolean algebra in which all the elements permute.

In the early development of structure theory for lattices, $L$ was assumed to be modular, and the notion of projectivity was used to study congruence relations. For non-modular lattices a more general concept was needed; accordingly, Dilworth [2] devised the notion of weak projectivity and showed that complementation has a strong influence on structure. He proved:

(1.3) Every relatively complemented lattice satisfying the ascending chain condition is the direct union of a finite number of simple relatively complemented lattices;

(1.4) Every finite dimensional locally relatively complemented lattice is a subdirect union of a finite union of simple, locally relatively complemented lattices;

(1.5) A relatively complemented lattice which satisfies a chain condition is simple if and only if all prime quotients are projective.

More recently these results have been developed and generalized by Tanaka [7], Maeda [6], and Hashimoto [4].

It is interesting to observe for the lattices described in (1.3), (1.4), and (1.5), weak projectivity of prime quotients reduces to projectivity. The present paper studies the relationship between weak projectivity and projectivity of prime quotients. It is shown that if $L$ satisfies the descending chain condition and if each join irreducible element of $L$ covers some element, then the corresponding irreducible congruence relations generate $\vartheta(L)$ and provide simple criteria for the structure of $L$.

2. Definitions. This section contains definitions of the basic terms which are used; terminology generally conforms to that given in Birkhoff [1].

Received May 4, 1959, and in revised form July 7, 1959. A major portion of this work was performed while the author was a National Science Foundation Faculty Fellow at Princeton University. 
(a) Quotients. If $a \supseteq b$ in $L$, the quotient $\{x \in L \mid b \subseteq x \subseteq a\}$ is denoted by $a / b$. If $a$ covers $b(a>b)$, then $a / b$ is called a prime quotient. The quotient $c / d$ is contained in $a / b$ if and only if $b \subseteq d \subseteq c \subseteq a$.

(b) Weak projectivity. A quotient $a / b$ is said to be weakly projective into a quotient $c / d(a / b$ WP $c / d)$ whenever there exists a finite sequence of quotients $a / b=x_{0} / y_{0}, x_{1} / y_{1}, \cdots, x_{k} / y_{k}=c / d$ such that $x_{i-1} / y_{i-1}$ is contained in a transpose of $x_{i} / y_{i}$. Weak projectivity of quotients is a reflexive and transitive relation, but, unlike projectivity, is not symmetric.

(c) Congruence relations. A congruence relation $\theta$ on $L$ is an equivalence relation which is preserved by the two basic lattice operations. Congruence relations are partially ordered by writing $\theta \subseteq \phi$ if and only if $a \equiv b(\theta)$ implies $a \equiv b(\phi)$. Under this ordering the set of all congruence relations on $L$ is a complete lattice $\vartheta(L)$ in which the operations are defined by

$a \equiv b\left(\cup_{s} \theta_{\alpha}\right)$ means $a=x_{0}, x_{1}, \cdots, x_{k}=b$ exist such that $x_{i-1} \equiv x_{i}$ $\left(\theta_{\alpha}\right)$ for some $\theta_{\alpha} \in S$,

$a \equiv b\left(\cap_{s} \theta_{\alpha}\right)$ means $a \equiv b\left(\theta_{\alpha}\right)$ for all $\theta_{\alpha} \in S$.

Furthermore, $\vartheta(L)$ is distributive (Funayama and Nakayama [3]). Two congruence relations are said to permute whenever $a \equiv c(\theta)$ and $c \equiv b(\phi)$ imply that $d$ exists such that $a \equiv d(\phi)$ and $d \equiv b(\theta)$. The center $\Gamma(L)$ of $\vartheta(L)$ is the set of all $\phi \in \vartheta(L)$ which permute with all $\theta \in \vartheta(L)$. The trivial congruence relations $c$ and $\omega$ are the unit and null elements of $\vartheta(L)$. The quotient $a / b$ is said to be collapsed by $\theta$ if and only if $a \equiv b(\theta)$. Clearly $x \equiv y(\theta)$ if and only if $(x \cup y) /(x \cap y)$ is collapsed by $\theta$. Then every quotient is collapsed by $\iota$, and no proper quotient is collapsed by $\omega$.

(d) Structural properties. $L$ is simple if and only if the congruence relations on $L$ are trivial. $L$ is irreducible if and only if there exist distinct elements $a$ and $b$ such that $a \equiv b(\theta)$ for every $\theta \neq \omega$. Simplicity implies irreducibility, but not conversely.

(e) Dimensionality. For the methods of this paper it is necessary to impose on $L$ the condition

( $\delta) ~ L$ satisfies the descending chain condition, and each join irreducible element covers some element.

Any such lattice will be called a $\delta$-lattice.

(f) Quotient ideals. Given a congruence relation $\theta$ on a lattice $L$, let $N(\theta)$ denote the set of all quotients collapsed by $\theta$. Then $N(\theta)$ is a quotient ideal as defined by Maeda [5]; that is, $N(\theta)$ satisfies

(2.1) $a / a \in N(\theta)$,

(2.2) if $a / b \in N(\theta)$ and $c / d \subseteq a / b$, then $c / d \in N(\theta)$,

(2.3) if $a / b \in N(\theta)$ and $a / b P c / d$, then $c / d \in N(\theta)$,

(2.4) if $a / b \in N(\theta)$ and $b / c \in N(\theta)$, then $a / c \in N(\theta)$.

Conversely, given any quotient ideal $N$, a congruence relation $\theta(N)$ is 
defined by writing $a \equiv b(\theta(N))$ if and only if $(a \cup b) /(a \cap b) \in N$. It follows that $N(\theta(N))=N$. Furthermore,

(2.5) $N(\theta) \subseteq N(\phi)$ if and only if $\theta \subseteq \phi$.

The connection between quotient ideals and weak projectivity is established as follows: let $S$ be any set of quotients of $L$, and denote by $N(S)$ the set of all quotients $a / b$ for which there exists a chain $a=x_{0} \supseteq x_{1} \supseteq \cdots \supseteq x_{k}=b$ such that $x_{i-1} / x_{i}$ is weakly projective into a quotient of $S$, for $i=1, \cdots, k$. Then $N(S)$ is the minimal quotient ideal containing $S$. In this way $S$ determines a congruence relation $\theta(S)=\theta(N(S))$ which is the minimal congruence relation which collapses the quotients of $S$.

This paper is concerned primarily with the case in which $S$ consists of a single irreducible prime quotient $q / c_{q}$, where $q$ is join irreducible and $q>c_{q}$; the corresponding congruence relation will be denoted by $\theta_{q}$. Such a relation will be called an irreducible congruence relation. This terminology is justified by the fact that precisely these congruence relations are the join irreducible elements of $\vartheta(L)$.

3. Irreducible congruence relations. Let $L$ be a $\delta$-lattice, and let $Q$ be the set of its join irreducible elements. For each $a \in L$ define

$$
Q(a)=\{q \in Q \mid q \subseteq a\} .
$$

Since the descending chain condition holds, each $a \in L$ is the union of a finite subset of $Q(a)$.

LEMma 3.1. If $a \supset b$, then $q / c_{q}$ WP $a / b$ for every $q \in Q(a)-Q(b)$, and $q / c_{q} \boldsymbol{P}(q \cup b) / b$ for every $q$ which is minimal in $Q(a)-Q(b)$.

Proof. If $q \in Q(a)-Q(b)$, then $q / c_{q} \subseteq q /(q \cap b) \boldsymbol{T}(q \cup b) / b \subseteq a / b$, so $q / c_{q} \boldsymbol{W P} a / b$. If $q$ is minimal in $Q(a)-Q(b)$, then $q \succ q \cap b$, and the second statement holds.

For each congruence relation $\theta$ on $L$ let $W(\theta)$ denote the set of all irreducibles $q$ for which $q / c_{q}$ is collapsed by $\theta$; that is,

$$
W(\theta)=\left\{q \in Q \mid q \equiv c_{q}(\theta)\right\}=\left\{q \in Q \mid q / c_{q} \in N(\theta)\right\} .
$$

Likewise for any quotient $a / b$, let $\theta(a / b)$ be the congruence relation generated by collapsing $a / b$; it follows that

$$
W(\theta(a / b))=\left\{q \in Q \mid q / c_{q} \boldsymbol{W P} a / b\right\},
$$

and we write $W(a / b)$ in place of $W(\theta(a / b))$. Using similar notation for projectivity, let 


$$
P(a / b)=\left\{q \in Q \mid q / c_{q} P a / b\right\} .
$$

The following statements are easy consequences of these definitions, the properties of projectivity and weak projectivity, and Lemma 3.1.

(3.1) The sets $P\left(q / c_{q}\right)$ for $q \in Q$ form a partition of $Q$.

(3.2) $P(a / b) \subseteq W(a / b)$.

(3.3) If $a / b$ is prime and if $q$ is minimal in $Q(a)-Q(b)$, then $P(a / b)=$ $P\left(q / c_{q}\right)$ and $W(a / b)=W\left(q / c_{q}\right)$.

(3.4) If $q \in W(a / b)$, then $P\left(q / c_{q}\right) \subseteq W\left(q / c_{q}\right) \subseteq W(a / b)$.

(3.5) $W(a / b)=\vee_{W(a / b)} P\left(q / c_{q}\right)$.

The remainder of this section is devoted to proving a sequence of lemmas concerning these sets, weak projectivity, and congruence relations to demonstrate the role of irreducible congruence relations in generating $\vartheta(L)$.

Lemma 3.2. If $a \supseteq b$, then $a \equiv b(\theta)$ if and only if $\theta(a / b) \subseteq \theta$.

Proof. Let $a \equiv b(\theta)$ and $x \equiv y(\theta(a / b))$. A chain, $x \cup y=a_{0} \supseteq a_{1}$ $\supseteq \cdots \supseteq a_{k}=x \cap y$, exists for which $a_{i-1} / a_{i} \boldsymbol{W P} a / b \in N(\theta)$. By (2.2), (2.3), and the definition of weak projectivity, $a_{i-1} / a_{i} \in N(\theta)$. Then $(x \cup y) /(x \cap y) \in$ $N(\theta)$ by (2.4), and $N(\theta(a / b)) \subseteq N(\theta)$. The lemma follows from (2.5), the reverse implication being trivial.

Corollary. $q \in W(\theta)$ if and only if $\theta_{q} \subseteq \theta$.

The next lemma is of fundamental importance, since it reveals that the collapse of any quotient can be accomplished by the collapse of a finite number of irreducible prime quotients; hence any congruence relation is a finite union of irreducible congruence relations.

LemMa 3.3. If $a \supset b$, there exists a finite set $S \subseteq Q(a)-Q(b)$ such that $a \equiv b\left(\cup_{s} \theta_{q}\right)$.

Proof. By the descending chain condition it may be assumed that every element properly contained in $a$ has the property asserted in the lemma. Let $S_{1} \subseteq Q(a)-Q(b)$ be chosen so that $q_{1} \in S_{1}$ is not redundant in the representation $a=b \cup \cup_{S_{1}} q$. Let $S_{2}=S_{1}-q_{1}$, and let $a_{2}=$ $b \cup \bigcup_{S_{2}} q$; then $a \supset a_{2} \supseteq b . \quad$ A finite set $S_{3} \subseteq Q\left(a_{2}\right)-Q(b)$ exists such that $a_{2} \equiv b \cup\left(\bigcup_{s_{3}} \theta_{q}\right)$. Also a finite set $S_{4} \subseteq Q\left(q_{1}\right)-Q\left(a_{2} \cap q_{1}\right)$ exists such that $q_{1} \equiv a_{2} \cap q_{1}\left(\mathrm{U}_{S_{4}} \theta_{q}\right)$. Then $S=S_{3} \vee S_{4}$ is a finite subset of $Q(a)-Q(b)$ for which $a \equiv b\left(\bigcup_{s} \theta_{q}\right)$.

LEMMA 3.4. If $a \supset b$, there exists a finite set $S \subseteq Q(a)-Q(b)$ such that $\theta(a / b)=\bigcup_{s} \theta_{q}$.

Proof. Lemmas 3.2 and 3.3 imply $\theta(a / b) \subseteq \bigcup_{s} \theta_{q}$. Conversely, 
Lemma 3.1, the definition of $W(\theta)$, and the Corollary imply $\theta_{q} \subseteq \theta(a / b)$ for every $q \in Q(a)-Q(b)$.

Lemma 3.5. For some finite set $S \subseteq W(\theta), \theta=\bigcup_{s} \theta_{q}$.

Proof. From the Corollary, $\bigcup_{W(\theta)} \theta_{q} \subseteq \theta$. Conversely, let $x \equiv y(\theta)$, $x \neq y$; by Lemma $3.3 x \cup y \equiv x \cap y\left(\bigcup_{s} \theta_{q}\right)$ for some finite $S \subseteq Q(x \cup y)-$ $Q(x \cap y)$. Hence $\theta \subseteq \bigcup_{s} \theta_{q}$. But $q / c_{q} \in N(x \cup y / x \cap y) \subseteq N(\theta)$ for every $q \in S$, so $S \subseteq W(\theta)$.

Lemma 3.6. $W(\theta) \subseteq W(\phi)$ if and only if $\theta \subseteq \phi$.

Proof. The direct implication follows from Lemma 3.5, while the reverse implication follows from (2.5) and the definition of $W(\theta)$. Observe that if equality holds in either relation, it holds in both.

LEMmA 3.7. $\theta$ is completely join irreducible in $\vartheta(L)$ if and only if $\theta=\theta_{q}$ for some $q \in Q$.

Proof. From Lemma 3.5 it is clear that any completely join irreducible $\theta$ must be of the form $\theta_{q}$ for some $q \in W(\theta) \subseteq Q$. Conversely, for any $q \in Q$ suppose $\theta_{q}=\bigcup_{\alpha \in A} \theta_{\alpha}$. Then $q \equiv c_{q}\left(\mathbf{U}_{\alpha \in A} \theta_{\alpha}\right)$, so there exists a finite sequence

$$
q=x_{0}, x_{1}, \cdots, x_{k}=c_{q}
$$

such that

$$
x_{i-1} \equiv x_{i}\left(\theta_{\alpha_{i}}\right), \text { for some } \alpha_{i} \in A,
$$

for $i=1,2, \cdots, k$. Then

$$
\left(x_{i-1} \cap q\right) \cup c_{q} \equiv\left(x_{i} \cap q\right) \cup c_{q}\left(\theta_{\alpha_{i}}\right) .
$$

But

$$
q \supseteq\left(x_{i} \cap q\right) \cup c_{q} \supseteq c_{q},
$$

so for each $i=0,1, \cdots, k,\left(x_{i} \cap q\right) \cup c_{q}$ equals $q$ or $c_{q}$. Since $q=\left(x_{0} \cap q\right) \cup c_{q}$ and $c_{q}=\left(x_{k} \cap q\right) \cup c_{q}$, there exists an index $j, 1 \leq j \leq k$, for which

$$
q=\left(x_{j-1} \cap q\right) \cup c_{q} \equiv\left(x_{j} \cap q\right) \cup c_{q}=c_{q}\left(\theta_{\alpha_{j}}\right) .
$$

By Lemma 3.2, $\theta_{q} \subseteq \theta_{\alpha_{j}}$; also the reverse relation holds by hypothesis, so $\theta_{q}=\theta_{\alpha_{j}}$.

Thus any completely irreducible element of $\vartheta(L)$ is an irreducible congruence relation, $\theta_{q}$, generated by collapsing an irreducible prime quotient of $L$. It follows from (3.3) and Lemma 3.3 that the collapse 
of any prime quotient generates an irreducible congruence relation. Clearly the number of distinct completely irreducible elements of $\vartheta(L)$ cannot exceed the number of distinct irreducibles in $L$. Two additional remarks concerning weak projectivity conclude this section.

LEMma 3.8. If $a / b$ WP $c / d$, then for each $q \in Q(a)-Q(b)$ there exists $\tilde{q} \in Q(c)-Q(d)$ such that $q / c_{q}$ WP $\tilde{q} / c_{q}$.

Proof. Let $q \in Q(a)-Q(b)$, where $a / b$ WP $c / d$. Then $q \in W(c / d)$, and $\theta_{q} \subseteq \theta(c / d)=\bigcup_{S} \theta_{\tilde{q}}$, where $S \subseteq Q(c)-Q(d)$. But in a distributive lattice if an irreducible is contained in the join of elements, it is contained in one of those elements. Hence $\theta_{q} \subseteq \theta_{\tilde{q}}$ for some $\tilde{q} \in S$. By Lemma 3.6, $q / c_{q}$ WP $\tilde{q} / c_{\tilde{q}}$.

Lemma 3.9. If $a / b$ WP $c / d$, then $\theta(a / b) \subseteq \theta(c / d)$; the converse holds if $a / b$ is prime.

Proof. If $a / b W \boldsymbol{P} c / d$, then $W(a / b) \subseteq W(c / d)$ since weak projectivity is transitive. By Lemma 3.6, $\theta(a / b) \subseteq \theta(c / d)$. Conversely, if $a / b$ is prime, then $a / b \boldsymbol{T} q / c_{q}$ for any minimal $q \in Q(a)-Q(b) \subseteq W(a / b)$. If also $\theta(a / b) \subseteq \theta(c / d), q \in W(c / d)$, so $a / b$ WP $c / d$.

4. Structure theorems. We now consider the role of irreducible congruence relations in determining the structure of $L$. From the theorems quoted in the introduction, it is clear that complementation in $\vartheta(L)$, permutability in $\vartheta(L)$, and the relation between weak projectivity of prime quotients have important effects on the structure of $L$.

THEOREM 4.1. In any $\delta$-lattice the following statements are equivalent :

(a) $\vartheta(L)$ is a Boolean algebra,

(b) for every $q \in Q, \theta_{q}>\omega$,

(c) the relation of weak projectivity is symmetric on the set of all irreducible prime quotients.

Proof. Any join irreducible element of a Boolean algebra must be a point, so (a) implies (b). Let $\theta_{q}$ be a point, and suppose $\tilde{q} / c_{\tilde{q}} \boldsymbol{W} \boldsymbol{P} q / c_{q}$. By Lemma 3.9, $\theta_{\tilde{q}} \subseteq \theta_{q}$, and equality must hold. Then $q / c_{q} \boldsymbol{W P} \tilde{q} / c_{\tilde{q}}$, again by Lemma 3.9 , so (b) implies (c). If weak projectivity is symmetric for all irreducible prime quotients, the sets $W\left(q / c_{q}\right)$ partition $Q$. For arbitrary $\theta$, let $\theta^{\prime}=\bigcup_{W^{\prime}(\theta)} \theta_{q}$, where $W^{\prime}(\theta)=Q-W(\theta)$. Then $\theta^{\prime}$ is a complement of $\theta$ in $\vartheta(L)$, which therefore is a Boolean algebra.

It follows from the preceding argument that $\vartheta(L)$ is a Boolean algebra if and only if the sets $W\left(q / c_{q}\right)$ partition $Q$. But also the sets 
$P\left(q / c_{q}\right)$ partition $Q$, and $P\left(q / c_{q}\right) \subseteq W\left(q / c_{q}\right)$. Thus if $\vartheta(L)$ is a Boolean algebra, the partition of $Q$ imposed by projectivity is a refinement of the partition imposed by weak projectivity. These two partitions can be distinct, even when $L$ is simple. However, weak projectivity of prime quotients does reduce to projectivity for a wide class of lattices - for example, modular lattices and the lattices described in (1.3), (1.4), and (1.5). In this connection the following theorem underlies corresponding results obtained by Dilworth [2] and Hashimoto [4] for relatively complemented lattices.

THEOREM 4.2. Let a $\delta$-lattice $L$ satisfy the condition that if two irreducible prime quotients are mutually weakly projective, then they are projective. Then $L$ is simple if and only if all prime quotients are projective.

Proof. Hashimoto uses the term uniserial to describe lattices in which all prime quotients are projective. Clearly any uniserial lattice is simple, because the collapse of any quotient collapses all of $L$. Conversely, if $L$ is simple, let $a / b$ and $c / d$ be prime quotients. By (3.3) and Lemma 3.6, $\theta(\alpha / b)=\theta\left(q / c_{q}\right)=\theta(c / d)=\theta\left(\tilde{q} / c_{\tilde{q}}\right)$ where the middle equality holds since $L$ is simple, and where $q$ and $\tilde{q}$ can be chosen to be minimal, respectively, in $Q(a)-Q(b)$ and $Q(c)-Q(d)$. Then $a / b \boldsymbol{T}$ $q / c_{q} \boldsymbol{W P} \tilde{q} / c_{\tilde{q}} \boldsymbol{T} c / d$, and $\tilde{q} / c_{\tilde{q}} \boldsymbol{W P} q / c_{q}$. Hence $q / c_{q} \boldsymbol{P} \tilde{q} / c_{\tilde{q}}$, and $\alpha / b \boldsymbol{P} c / d$.

THeorem 4.3. If $L$ is a $\delta$-lattice for which $\vartheta(L)$ is a Boolean algebra, then $L$ is simple if and only if $L$ is irreducible.

Proof. Let $a \neq b$ be elements which establish the irreducibility of $L ; a \equiv b(\theta)$ for all $\theta \neq \omega$. For all $q \in Q, a \equiv b\left(\theta_{q}\right)$; thus $\theta(a \cup b / a \cap b) \subseteq \theta_{q}$. Therefore, for some $\tilde{q}$ and all $q, \theta(a \cup b / a \cap b)=\theta_{\tilde{q}} \subseteq \theta_{q}$, so $\vartheta(L)$ has $\theta_{\tilde{q}}$ as its only point. But if $\vartheta(L)$ is also a Boolean algebra, $\theta_{\tilde{q}}=\theta_{q}$ for all $q$, and therefore $L$ is simple. The converse is well known.

Corollary. A s-lattice $L$ is irreducible if and only if $\vartheta(L)$ has a single point.

Proof. The preceding proof shows that $\vartheta(L)$ has a unique point if $L$ is irreducible. But if $\theta_{q}$ is the only point of $\vartheta(L)$, then $\theta \neq \omega$ implies $\theta_{q} \subseteq \theta$, and thus $q \equiv c_{q}(\theta)$. Therefore $q$ and $c_{q}$ satisfy the condition of irreducibility for $L$.

Our remaining remarks concern complementation and permutability of irreducible congruence relations. The investigation of these properties arises naturally because any direct decomposition of $L$ determines a congruence relation $\theta$ which has a complement and which permutes 
with all congruence relations. A congruence relation with these two properties is called a decomposition congruence relation, and the set of all decomposition congruence relations forms a Boolean sublattice of $\vartheta(L)$.

LEMma 4.1. $\theta_{q}$ has a complement in $\vartheta(L)$ if and only if $\theta_{q}$ satisfies the condition that $\tilde{q} \in Q$ and $\theta_{q} \cap \theta_{\tilde{q}} \neq \omega$ imply $\theta_{\tilde{q}} \subseteq \theta_{q}{ }^{1}$

Proof. Let $\theta^{*}$ be a complement of $\theta_{q}$; it is easily verified that $W\left(\theta^{*}\right)$ is the complement of $W\left(\theta_{q}\right)$ in $Q$. Let $\theta_{q} \cap \theta_{\tilde{q}}=\theta \supset \omega$. Then $W\left(\theta_{q}\right) \supseteq W(\theta)$ so $W(\theta) \wedge W\left(\theta^{*}\right)$ is void. Also $W(\theta) \subseteq W\left(\theta_{\tilde{q}}\right)$, so if $\tilde{q} \in W\left(\theta^{*}\right)$, then by the Corollary following Lemma 3.2, $W(\theta) \subseteq W\left(\theta_{\tilde{q}}\right) \subseteq$ $W\left(\theta^{*}\right)$, which is a contradiction. Hence $\tilde{q} \in W\left(\theta_{q}\right)$ and $\theta_{\tilde{q}} \subseteq \theta_{q}$. Conversely, suppose $\theta_{q}$ satisfies the condition stated in the lemma. Lat $W^{*}=Q-W\left(\theta_{q}\right)$, and let $\theta^{*}=\bigcup_{W^{*}} \theta_{\tilde{q}}$. Then $W\left(\theta^{*}\right) \supseteq W^{*}$, so $\theta_{q} \cup \theta^{*}=\iota$; and $\theta_{q} \cap \theta^{*}=\bigcup_{W^{*}}\left(\theta_{q} \cap \theta_{\tilde{q}}\right)$. But $\tilde{q} \in W^{*}$ implies $\theta_{\tilde{q}} \nsubseteq \theta_{q}$, so $\theta_{q} \cap \theta_{\tilde{q}}=\omega$ for all $\tilde{q} \in W^{*}$. Thus $\theta_{q} \cap \theta^{*}=\omega$.

THEOREM 4.4. If $L$ is a $\delta$-lattice, then $\vartheta(L)$ is a Boolean algebra if and only if $\theta_{q}$ has a complement for every $q \in Q$.

Proof. The condition is trivially necessary. Suppose each $\theta_{q}$ has a complement; then Lemma 4.1 implies that if $\theta_{\bar{q}} \cap \theta_{\tilde{q}} \neq \omega$, then $\theta_{\bar{q}} \subseteq \theta_{\tilde{q}}$ and $\theta_{\tilde{q}} \subseteq \theta_{\bar{q}}$. Hence for each $q \in Q, \theta_{q}$ must be a point, so by Theorem $4.1 \vartheta(L)$ is a Boolean algebra.

Theorem 4.5. If $L$ is a $\delta$-lattice, then $\Gamma(L)=\vartheta(L)$ if and only if $\theta_{q}$ and $\theta_{\tilde{q}}$ permute for all $q, \tilde{q} \in Q$.

Proof. The necessity is trivial; the sufficiency follows from Lemma 3.5 and the fact that if $\theta$ permutes with each member of a set of congruence relations, then $\theta$ permutes with any union of them.

Combining Theorems 4.4 and 4.5 with (1.1) and (1.2), we see that under suitable dimensionality conditions, $L$ is a subdirect union of simple lattices if and only if each $\theta_{q}$ has a complement, while $L$ is a direct union of simple lattices if and only if each $\theta_{q}$ is a decomposition congruence relation.

\section{BIBLIOGRAPHY}

1. G. Birkhoff, Lattice Theory, Rev. ed. Amer. Math. Soc. Colloquium Publications, Vol. 25 (1948).

2. R. P. Dilworth, The structure of relatively complemented lattices. Annals of Math, 51,

1 As the referee has pointed out, Lemma 4.1 expresses a property of the join irreducibles of any complete, distributive lattice which satisfies the descending chain condition. 
No. 2 (1950), 348-359.

3. N. Funayama, and T. Takayama, On the distributivity of a lattice of congruence relations. Proc. Imp. Acad. Tokyo, 18 (1942), 553-554.

4. J. Hashimoto, Direct, subdirect decompositions and congruence relations. Osaka Math. J., 9 (1957), 87-112.

5. F. Maeda, Kontinuierliche Geometrien, Grundlehren der Math. Wiss., Band XCV (1958).

6. - Direct and subdirect factorizations of lattices, J. Sci. Hiroshima Univ., Ser.

A, 15 (1951), 99-102.

7. T. Tanaka, Canonical subdirect factorizations of lattices, J. Sci. Hiroshima Univ., Ser. A, 16 (1952), 239-246.

Kenyon College 



\section{PACIFIC JOURNAL OF MATHEMATICS}

\section{EDITORS}

\author{
David Gilbarg \\ Stanford University \\ Stanford, California \\ F. H. Brownell \\ University of Washington \\ Seattle 5 , Washington
}

\author{
A. L. Whiteman \\ University of Southern California \\ Los Angeles 7, California \\ L. J. PAIGE \\ University of California \\ Los Angeles 24, California
}

\section{ASSOCIATE EDITORS}

\author{
E. F. BECKENBACH \\ T. M. CHERRY \\ D. DERRY
}
E. HEWITT
A. HORN
L. NACHBIN
M. OHTSUKA
H. L. ROYDEN
M. M. SCHIFFER

E. SPANIER

E. G. STRAUS

F. WOLF

\section{SUPPORTING INSTITUTIONS}

\author{
UNIVERSITY OF BRITISH COLUMBIA \\ CALIFORNIA INSTITUTE OF TECHNOLOGY \\ UNIVERSITY OF CALIFORNIA \\ MONTANA STATE UNIVERSITY \\ UNIVERSITY OF NEVADA \\ NEW MEXICO STATE UNIVERSITY \\ OREGON STATE COLLEGE \\ UNIVERSITY OF OREGON \\ OSAKA UNIVERSITY \\ UNIVERSITY OF SOUTHERN CALIFORNIA
}

\author{
STANFORD UNIVERSITY \\ UNIVERSITY OF TOKYO \\ UNIVERSITY OF UTAH \\ WASHINGTON STATE COLLEGE \\ UNIVERSITY OF WASHINGTON

AMERICAN MATHEMATICAL SOCIETY
CALIFORNIA RESEARCH CORPORATION
HUGHES AIRCRAFT COMPANY
SPACE TECHNOLOGY LABORATORIES
NAVAL ORDNANCE TEST STATION

Mathematical papers intended for publication in the Pacific Journal of Mathematics should be typewritten (double spaced), and the author should keep a complete copy. Manuscripts may be sent to any one of the four editors. All other communications to the editors should be addressed to the managing editor, L. J. Paige at the University of California, Los Angeles 24, California. ..

50 reprints per author of each article are furnished free of charge; additional copies may be obtained at cost in multiples of 50 .

The Pacific Journal of Mathematics is published quarterly, in March, June, September, and December. The price per volume (4 numbers) is $\$ 12.00$; single issues, $\$ 3.50$. Back numbers are available. Special price to individual faculty members of supporting institutions and to individual members of the American Mathematical Society: $\$ 4.00$ per volume; single issues, $\$ 1.25$.

Subscriptions, orders for back numbers, and changes of address should be sent to Pacific Journal of Mathematics, 2120 Oxford Street, Berkeley 4, California.

Printed at Kokusai Bunken Insatsusha (International Academic Printing Co., Ltd.), No. $6_{4}$ 2-chome, Fujimi-cho, Chiyoda-ku, Tokyo, Japan.

PUBLISHED BY PACIFIC JOURNAL OF MATHEMATICS, A NON-PROFIT CORPORATION

The Supporting Institutions listed above contribute to the cost of publication of this Journăl, but they are not owners or publishers and have no responsibility for its content or policies. 


\section{Pacific Journal of Mathematics}

\section{Vol. 10, No. $3 \quad$ November, 1960}

Glen Earl Baxter, An analytic problem whose solution follows from a simple

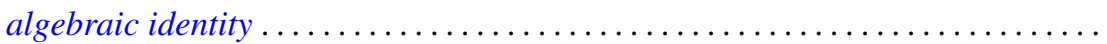

Leonard D. Berkovitz and Melvin Dresher, A multimove infinite game with linear payoff. .

Earl Robert Berkson, Sequel to a paper of A. E. Taylor ......................

Gerald Berman and Robert Jerome Silverman, Embedding of algebraic systems.... 767

Peter Crawley, Lattices whose congruences form a boolean algebra . . . . . ...... 777

Robert E. Edwards, Integral bases in inductive limit spaces . . . . . . . . . . . . . . .

Daniel T. Finkbeiner, II, Irreducible congruence relations on lattices . . . . . . . . . .

William James Firey, Isoperimetric ratios of Reuleaux polygons . . . . . . . . . . . 787

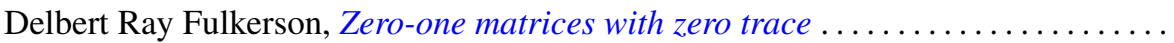

Leon W. Green, A sphere characterization related to Blaschke's conjecture........

Israel (Yitzchak) Nathan Herstein and Erwin Kleinfeld, Lie mappings in

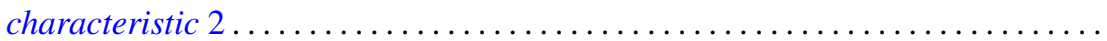

Charles Ray Hobby, A characteristic subgroup of a $p$-group .................

R. K. Juberg, On the Dirichlet problem for certain higher order parabolic

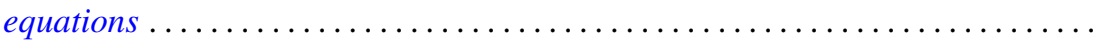

Melvin Katz, Infinitely repeatable games ......................

Emma Lehmer, On Jacobi functions . . . . . . . . . . . . . . . . . . . . . . . . .

D. H. Lehmer, Power character matrices

Henry B. Mann, A refinement of the fundamental theorem on the density of the sum of two sets of integers.

Marvin David Marcus and Roy Westwick, Linear maps on skew symmetric matrices: the invariance of elementary symmetric functions . .

Richard Dean Mayer and Richard Scott Pierce, Boolean algebras with ordered

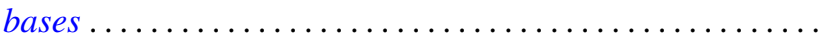

Trevor James McMinn, On the line segments of a convex surface in $E_{3} \ldots$

Frank Albert Raymond, The end point compactification of manifolds ..

Edgar Reich and S. E. Warschawski, On canonical conformal maps of regions of arbitrary connectivity

Marvin Rosenblum, The absolute continuity of Toeplitz's matrices...

Lee Albert Rubel, Maximal means and Tauberian theorems . .

Helmut Heinrich Schaefer, Some spectral properties of positive linear operators

Jeremiah Milton Stark, Minimum problems in the theory of pseudo-conformal transformations and their application to estimation of the curvature of the invariant metric.

Robert Steinberg, The simplicity of certain groups ...

Hisahiro Tamano, On paracompactness. .

Angus E. Taylor, Mittag-Leffler expansions and spectral theory .

Marion Franklin Tinsley, Permanents of cyclic matrices ...... . 